\title{
Agricultural Robots: A Different Approach
}

\author{
Arnold Flavious D'souza ${ }^{1}$, Ankith Rai ${ }^{2}$, Aster Fernandes ${ }^{3}$, Jolene Shaina Vas ${ }^{4}$, Mr. Vaishak N.L ${ }^{5}$ \\ Student, $4^{\text {th }}$ Year, Mechanical Engineering, SCEM, Adyar, Mangalore ${ }^{1,2,3,4}$ \\ Assistant Professor, Department of Mechanical Engineering, SCEM, Adyar, Mangalore ${ }^{5}$
}

\begin{abstract}
The main objective is to develop a model capable of performing multiple farming operations by incorporating a number of agricultural equipment into an autonomous robot. It contains a hopper, harvester, plougher, medicine sprinkler, fertilizer sprayer and chain drive mechanism. These components are controlled by microcontrollers and this is in turn operated by remote control.The approach is now to develop smarter machines that are intelligent enough to work in an unmodified or semi natural environment such as paddy or wheat fields. These machines do not have to be intelligent in the way we see people as intelligent but must exhibit sensible behaviour in recognised contexts. A single machine carrying out a variety of operations will greatly reduce costs of operations. Fertilizers are remotely dispersed which reduces the exposure of these chemicals to humans. Huge tracts of land can be cultivated in a short amount of time. Since the operations are automated, costs of employing humans are reduced.
\end{abstract}

Keywords: Agribot, Plougher, Harvester, Hopper.

\section{INTRODUCTION}

The idea of applying robotics technology in agriculture is himself, such as controlling the fields for pests. These very new. In agriculture, the opportunities for robot- tasks are perfectly suited for autonomous robots, as they enhanced productivity are immense and robots are often require numerous repetitions over a long period of appearing on farms in various guises and in increasing time and over a large area. The use of robots is a rather numbers. We can expect the robots to perform agricultural new development as most of the existing solutions for operations autonomously such as spraying and mechanical automatic supervision, is designed for standard farm weed control, fruit picking, watching the farms day \& equipment such as tractors, combines and pesticide night for an effective report, allowing farmers to reduce sprayers.

the environmental impact, increase precision and In most cases a small agricultural robot would be efficiency, and manage individual plants in novel ways. ineffective in performing farming jobs, as these often The applications of instrumental robotics are spreading require a large quantity of materials, either to put into the every day to cover further domains, as the opportunity of ground, such as seeds or fertilisers, or to take from the replacing human operators provides effective solutions field during harvest. But when dealing with monitoring with return on investment. Farming requires a variety of and mapping of fields or precision spraying of pesticides, processes to be carried out in order to obtain the required a smaller robot is ideal, as it is gentler on the crops but yield. These processes may vary from ploughing the land, also to the ground. This is due to the lower weight sowing of seeds, spraying of fertilizers and medicines etc. compared to a tractor, causing much lesser soil Presently, a variety of machines are required to carry out compaction. The degree of soil compaction is important to these processes which can be expensive to operate and consider, especially when dealing with monitoring and maintain. To resolve this issue, a single piece of automated mapping as this is often performed multiple times machinery (AGRIBOT) which can perform a variety of throughout the year, as soil compaction can cause a operations can be developed. This machine will carry a number of problems, such as reduced crop growth and deploughing mechanism, hopper, medicine and fertilizer nitrification.

sprayer as well as a harvester operated remotely. The approach of treating crop and soil selectively Therefore, an introduction of such machinery would according to their needs by small autonomous machines is greatly reduce time and resources with an increase in the natural next step in the development of Precision efficiency.

\section{LITERATURE}

As farms grow in size, together with the size of the equipment used on them, there is a need for ways to automate processes, previously performed by the farmer

Farming (PF) as it reduces the field scale right down to the individual plant or Phytotechnology (Shibusawa 1996). One simple definition of PF is doing the right thing in the right place at the right time with the right amount. This definition not only applies to robotic agriculture (RA) and Phytotechnology but it also implies a level of automation inherent in the machines. Automatic sensing and control 


\author{
National Conference on Advances in Electrical Engineering \\ NMAM Institute of Technology, Nitte \\ Vol. 5, Special Issue 2, April 2017
}

(on-the-go) for each task is also important and many research papers have shown that these systems are feasible but most are too slow, and hence not economically viable, to be operated on a manned tractor. Once these systems are mounted on an autonomous vehicle, they may well suddenly become commercially viable.

Modern agriculture uses a lot of energy. It comes in many forms from fertilisers andchemicals to tractors and fuel. The Phytotechnology approach tries to target the introduced energy to improve efficacy. Chamen (1994) identified that a $70 \%$ energy saving can be made in cultivation energy by moving from traditional trafficked systems (255 MJ/ha) to a non-trafficked system (79 $\mathrm{MJ} / \mathrm{ha}$ ). This was for shallow ploughing and did not include any deep loosening. From this we estimate that 80$90 \%$ of the energy going into traditional cultivation is there to repair the damage done by large tractors. It would be much better to not cause compaction in the first place which is one of the reasons that leads us to consider using small light machines.

Conventional techniques depend on human power for lifting, dragging, weed control, fruit picking. Humans are prone to work in hazardous environment while spraying chemicals and pesticides. The tractors compact the soil, as they are larger in weight. They cannot move in terrain conditions. These methods cannot identify the crop and soil in close proximity. In the case of automated agriculture (which uses field robots) is exemplified from above problems. Robots can work tirelessly in all environments; all you have to do is set a program to perform the desired activities.

Although, large sized wheels are required in muddy soils, robots with small sized wheels perform well. Robot scouts are employed to get detailed information about the crop such as the presence of diseases, weeds, insect infestations and other stress conditions. The lightweight of the robots is a major advantage, since they do not compact the soil as larger machinery does.

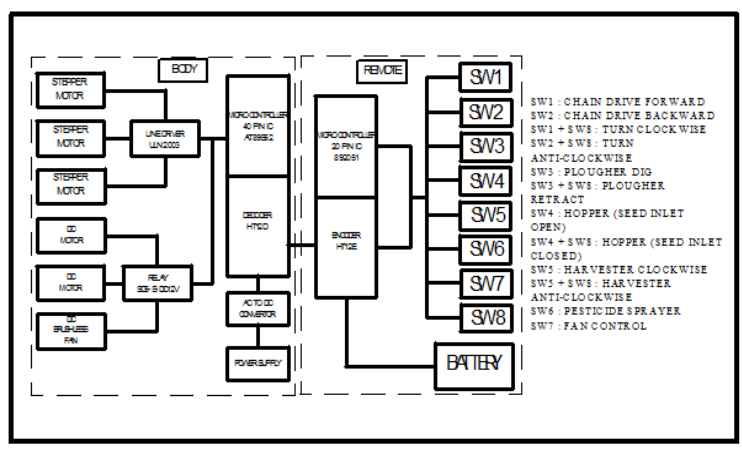

Fig 1: Block Diagram of the System

\section{III.METHODOLOGY ADOPTED}

An Agribot is a multipurpose machine which helps to simplify the work of the farmer. It has five functions in it which helps in to improving the yield of the crop and reduces the work hours and man hours put into it.The cycle of agriculture is ploughing, sowing seeds, fertilizers, medicine which is followed by harvesting. These cycles are performed by a single machine. The main components of the Agribot are as follows:

\section{A. Hopper}

The hopper is storage material used to store the seeds and generally a guiding unit which segregates the seed in the correct order into the ground. This generally helps out for uniform distribution of seed into the ground.

The material used for this design of low capacity robots is sheet metal whereas for further improvement it can be made of much heavier materials for effective mechanization on agricultural robot

\section{B. Plougher}

Plougher is a unit which help in opening up the ground and its minerals which is to reach the proteins and minerals which are lodged inside the ground. Plougher helps to open up the ground and helps the seed to get in and also helps in mixing up the natural humus and fertilisers. The primary purpose of ploughing is to turn over the upper layer of the soil, bringing fresh nutrients to the surface, while burying weeds, the remains of previous crops, and both crop and weed seeds, allowing them to break down. It also aerates the soil, allows it to hold moisture better and provides a seed-free medium for planting an alternate crop. In modern use, a ploughed field is typically left to dry out, and is then harrowed before planting.

\section{Harvester}

The combine harvester, or simply combine, is a machine that harvests grain crops. The name derives from its combining three separate operations comprising of harvesting, reaping, threshing, and winnowing into a single process. Among the crops harvested with a combine are wheat, oats, rye, barley, corn (maize), soybeans and flax (linseed). The waste straw left behind on the field is the remaining dried stems and leaves of the crop with limited nutrients which is either chopped and spread on the field or baled for feed and bedding for livestock.

\section{Chain/Tracks}

Chain/ tracks are a system of vehicle propulsion in which a continuous band of treads is driven by two or more wheels. This band is typically made of modular steel plates in the case of military vehicles, or rubber reinforced with steel wires in the case of lighter agricultural or construction vehicles. The large surface area of the tracks distributes the weight of the vehicle better than steel or rubber tires on an equivalent vehicle, enabling a continuous tracked vehicle to traverse soft ground with less likelihood of becoming stuck due to sinking. The 


\title{
International Journal of Innovative Research in Electrical, Electronics, Instrumentation and Control Engineering NCAEE 2017
}

\author{
National Conference on Advances in Electrical Engineering \\ NMAM Institute of Technology, Nitte \\ Vol. 5, Special Issue 2, April 2017
}

prominent treads of the metal plates are both hard-wearing decoded when no error or unmatched codes are found. A and damage resistant, especially in comparison to rubber valid transmission in indicated by a high signal at VT pin. tires.

\section{E. Main Body Frame}

It is the main housing for all the attachments and other holding devices. A robotic body should possess a very versatile body to resist all the bearing forces and should have a simple structure.

\section{F. Microcontrollers}

A microcontroller is a small computer on a single integrated circuit containing a processor core, memory, and programmable input/output peripherals.

For the body, a microcontroller 40 PIN IC AT89S52 is used. The AT89S52 is a low-power, high-performance CMOS 8-bit microcontroller with $8 \mathrm{~K}$ bytes of in-system programmable Flash memory. The device is manufactured using Atmel's high-density non-volatile memory technology and is compatible with the industry-standard 80C51 instruction set.

For the remote, a microcontroller 20 PIN IC AT89C2051 is used. The AT89C2051 is a low-voltage, highperformance CMOS 8-bit microcomputer with $2 \mathrm{~K}$ bytes of Flash programmable and erasable read-only memory (PEROM). The device is manufactured using Atmel's high-density non-volatile memory technology and is compatible with the industry-standard MCS-51 instruction set. By combining a versatile 8-bit CPU with Flash on a monolithic chip, the Atmel AT89C2051 is a powerful microcomputer which provides a highly-flexible and costeffective solution to many embedded control applications.

\section{G. Remote Encoder and Body Decoder}

In the remote, a HT12E encoder is used. HT12E is an encoder integrated circuit of 212 series of encoders. They are paired with 212 series of decoders for use in remote control system applications. It is mainly used in interfacing RF and infrared circuits. The chosen pair of encoder/decoder should have same number of addresses and data format. Simply put, HT12E converts the parallel inputs into serial output. It encodes the 12 bit parallel data into serial for transmission through an RF transmitter. These 12 bits are divided into 8 address bits and 4 data bits.

In the body, a HT12D decoder is used.HT12D is a decoder integrated circuit that belongs to 212 series of decoders. This series of decoders are mainly used for remote control system applications, like burglar alarm, car door controller, security system etc.In simple terms, HT12D converts the serial input into parallel outputs. It decodes the serial addresses and data received by, say, an RF receiver, into parallel data and sends them to output data pins. The serial input data is compared with the local addresses three times continuously. The input data code is

\section{H. Line Driver}

ULN2003 is the line driver that is employed. ULN2003 is a high voltage and high current Darlington array IC. It contains seven open collector Darlington pairs with common emitters. A Darlington pair is an arrangement of two bipolar transistors. It belongs to the family of ULN200X series of ICs. Different versions of this family interface to different logic families. ULN2003 is for $5 \mathrm{~V}$ TTL, CMOS logic devices. These ICs are used when driving a wide range of loads and are used as relay drivers, display drivers, line drivers etc.

\section{Stepper Motor}

The stepper motor is an electromagnetic device that converts digital pulses into mechanical shaft rotation. Advantages of step motors are low cost, high reliability, high torque at low speeds and a simple, rugged construction that operates in almost any environment. The main disadvantages in using a stepper motor is the resonance effect often exhibited at low speeds and decreasing torque with increasing speed.

\section{J. Power Source}

A 20 Watt solar panel is used as a power source for the Agribot. The solar panel is connected to a solar charge controller. This product can automatically manage the work of the solar panel and battery in the solar setup, which is below 120 Watt (12V). The solar charge controller is connected to the battery as well as the Agribot which requires a $12 \mathrm{~V}$ Direct Current as an input.

\section{K. Working:}

First is the ploughing cycle, in which the Agribot uses a plougher attachment to open the ground to open up the minerals and proteins inside the ground. It accelerates the soil warming as well as water evaporation. Also due to ploughing, it increases the soil rotation and aides in farming. The seeds are stored in a hopper in the Agribot which helps the seeds to be segregated in the order which helps in easy distribution. The seed are sent through a channel to the distributing fan. This is operated through an open and close door opening system which opens and closes the door at the required time and the right amount.

Next is the fertilizer sprinkler which operates on command. This is used to improve the proteins and nutrients of the soil in the field, this boost the yield of the crops which is also done automatically with a button to control it. A water sprinkler is also used to moist the earth during ploughing which requires a considerable amount of water. Next, a medicine sprinkler is used and it helps the plant to grow in a healthy manner and produce good yield at the end of the cycle. It also includes spraying pesticides to avoid infestation of the plants, reduces the loss of crops 
National Conference on Advances in Electrical Engineering

NMAM Institute of Technology, Nitte

Vol. 5, Special Issue 2, April 2017

due to worms and other insects, thereby increasing the crop life. Harvesting is a part of the process which gathers the mature crops from the field. Gathering crops from the field with the help of an autonomous robot proves to be highly efficient when compared to manual labour. This process also includes cutting the crops to the required length and then separating the husk from the crop by removing the unwanted parts of the plant. The husks are then discarded and the crops are bagged and stored. The robot can be powered by employing an eco-friendly source of power such as solar energy.

\section{IV.LIMITATIONS}

Agribots are a relatively new concept. As true with everything, new concepts require time and research to perfect the technology. Though the concept of automated farming is appealing, the access to this new technology is limited. Extensive research and field work is required before this technology can be perfected to a degree with which the technology can be purchased off the shelf. Also, as this new technology is yet to blossom into an everyday need into farming, limited production and the research and development prove these machines to be expensive to purchase and maintain. Thirdly, considering the future scope for development, incorporation of sensors would be required. These sensors would be essential to detect factors such as moisture level in the soil, rate of harvesting etc. Incorporation of multiple sensors onto the Agribot is a complex task. With robots, the question of liability also arises. Finally, farming is a traditional process wherein the methods used are deeply rooted in the culture of the land. Willingness to accept a renewed style of farming and doing away with the old methods may prove to be a difficult task to a significant portion of the farming community.

\section{CONCLUSION}

Automation increases efficiency. An efficient process results in greater yield and productivity which corresponds to lower costs and higher profits. The problems associated with the proposed model can be overcome with technology in time. Autonomous farming may redefine how crop production is done by phasing out larger machines. It may be wise to consider that crop production may be done better and economically with a swarm of small machines than with a few large ones.One of the advantages of autonomous farming may be employment to the traditionally non-farming community. The jobs in agriculture are arduous and labour intensive but require repetitive decisions and hence robots can be rightly substituted with humans in most cases. The higher quality products can be sensed by machines (colour. firmness, weight, density, ripeness, size, shape) accurately. Robots can prove to be a great boon to agriculture.

\section{REFERENCES}

[1] NarzuTarannum, Md. KhalilurRhaman, Sabbir Ahmed Khan, ShifurRahmanShakil, "A Brief Overview and Systematic Approch for Using Agricultural Robot in Developing Countries", Journal of Modern Science and Technology Vol. 3. No.1. March 2015. Issue. Pp. 88-101.

[2] M. priyadarshini, Mrs. L. Sheela, "Command based self-guided digging and seed sowing rover",International Conference on Engineering Trends and Science \& Humanities, ISSN: 2348 - 8379, ICETSH-2015.

[3] S. Blackmore, B. Stout, M. Wang, and B. Runov, Robotic agriculture - The futureof agricultural mechanisation?" 5th Eur. Conf. Precis. Agric. (ECPA), Upsala, no.June 2005, pp. 621-628, 2005.

[4] A. Singh, A. Gupta, A. Bhosale, and S. Poddar, Agribot: - An Agriculture Robot, IJARCCE, 4(1), pp. 317-319, 2015.

[5] R. Lenain, B. Thuilot, C. Cariou, and P. Martinet, High accuracy path tracking for vehicles in presence of sliding: Application to farm vehicle automatic guidance for agricultural tasks, Auton. Robots, 21(1), pp. 79-97, 2006.

[6] H. W. Griepentrog, M. Norremark, H. Nielsen, and B. S. Blackmore, Seed mapping of sugar beet, Precis. Agric., 6(2), pp. 157-165, 2005.

[7] S. M. Pedersen, S. Fountas, and S. Blackmore, Agricultural Robots -Applications and Economic Perspectives, Serv. Robot Appl., no. August, 2008.

[8] QingchunFeng,Xiaonan Wang, Guohua Wang, Zhen Li, “Design and Test of Tomatoes Harvesting Robot," International Conference on Information and Automation Lijiang, China, pp 949-952, IEEE 2015

[9] AkashBhosale and SumeetPoddar published a paper on "An autonomous robot for harvesting cucumbers in green houses" (IEEE- 2011).

[10] Bill Stout and Maohua Wang published a paper on "Detecting tomato crops in green houses using a vision based system" (IEEE2012).

[11] Graglia published a paper on "Autonomous robots for agricultural tasks and farm assignment and future trends in agro robots" (IJMME-IJNS-2013)

[12] Weather head published a paper on "An autonomous tree climbing robot utilizing four bar linkage system" (CIGR-2002).

[13] K.V.Fale and P.Bhureamit published a paper on "Autonomous farming robot with plant health indication" (IJATES-2015)

[14] Ankit Singh and Abhishek Gupta published a paper on "agribot" (IJARCCE-2015). 